\title{
Ranking authors using fractional counting of citations: An axiomatic approach ${ }^{1}$
}

\author{
Denis Bouyssou $^{2} \quad$ Thierry Marchant ${ }^{3}$
}

15 December 2015

\begin{abstract}
This paper analyzes from an axiomatic point of view a recent proposal for counting citations: the value of a citation given by a paper is inversely proportional to the total number of papers it cites. This way of fractionally counting citations was suggested as a possible way to normalize citation counts between fields of research having different citation cultures. It belongs to the "citing-side" approach to normalization. We focus on the properties characterizing this way of counting citations when it comes to ranking authors. Our analysis is conducted within a formal framework that is more complex but also more realistic than the one usually adopted in most axiomatic analyses of this kind.
\end{abstract}

Keywords: bibliometric ranking of authors, field normalization, fractional counting of citations, axiomatization.

\section{Introduction}

With the increasing use of bibliometric indices for evaluation and monitoring purposes, there are many situations in which one has to compare papers, authors,

Authors are listed in alphabetical order. They have equally contributed to the paper.

${ }^{1}$ We thank Michel Zitt for his comments on an earlier draft of this text. We are most grateful to Ludo Waltman for his detailed comments and his very useful pointers to the literature. Two anonymous referees of this journal made useful comments. The usual caveat applies.

${ }^{2}$ CNRS (LAMSADE, UMR 7243) \& Université Paris Dauphine, Place du Maréchal de Lattre de Tassigny, F-75 775 Paris Cedex 16, France, tel: +331 440548 98, fax: +331440540 91, e-mail: bouyssou@lamsade.dauphine.fr.

${ }^{3}$ Ghent University, Department of Data Analysis, H. Dunantlaan, 1, B-9000 Gent, Belgium, e-mail: thierry.marchant@UGent.be. 
research units, journals belonging to different fields of research (see, e.g., RuizCastillo and Costas, 2014). But each field of research has its own "citation culture". Without trying to be exhaustive, elements of the culture of a field include: i) its more or less complete coverage by bibliometric database, ii) its growth rate, iii) its tendency to import citations from or to export citations to other fields, $i v)$ its size, $v$ ) its publication intensity, i.e., the typical number of papers published per year, vi) its citation intensity, i.e., the typical length of the list of references in a publication, vii) the tendency to publish research in other outlets than peerreviewed journals, viii) the existence of journals specialized in publishing review papers, $i x)$ its co-authorship practices, $x$ ) the length of the period during which a paper published is usually cited.

This makes the problem of "normalization" between fields a difficult but important challenge: it is well-known that comparing citations counts in Mathematics with citations counts in Biology is inherently difficult (Amin and Mabe, 2000). Although this is an old theme (Schubert and Braun, 1986, 1996) in the bibliometric literature, there has been a recent increase in the literature devoted to this question. Two main paths have been followed to tackle the question ${ }^{1}$.

The first path (sometimes called "a posteriori" normalization or "cited-side" normalization) consists in comparing citation counts with what is observed (usually, on average) in the field. This requires to have at hand a delineation of fields. This is not as obvious as it may seem at first sight (see, e.g., van Raan, 2004, 2005, Small and Sweeney, 1985, Waltman and van Eck, 2012a, Waltman, van Eck, and Noyons, 2010). For instance, some papers (authors, journals) will be at the border between two fields. Moreover, the adequate granularity of this partition into fields is not easy to determine. Even worse, important differences may exist within what is usually considered to be a field (van Eck, Waltman, van Raan, Klautz, and Peul, 2013, Franceschini and Maisano, 2014). This line of research has recently been revived by the debate about "universality of citations distributions" (Radicchi, Fortunato, and Castellano, 2008, Waltman, van Eck, and van Raan, 2012b) and has generated numerous empirical studies (Abramo, Cicero, and D'Angelo, 2012, Albarrán, Crespo, Ortuño, and Ruiz-Castillo, 2011, Bornmann and Daniel, 2009, Crespo, Li, and Ruiz-Castillo, 2013, Crespo, Herranz, Li, and Ruiz-Castillo, 2014, Li and Ruiz-Castillo, 2013, Li, Radicchi, Castellano, and Ruiz-Castillo, 2013, Radicchi and Castellano, 2012b, Ruiz-Castillo, 2012, RuizCastillo and Waltman, 2015) (see also the debate around the "crown indicator" of

\footnotetext{
${ }^{1}$ As pointed out to us by a referee, the work of Kaur, Ferrara, Menczer, Flammini, and Radicchi (2015), extending Crespo, Ortuño-Ortín, and Ruiz-Castillo (2012), can be seen as a third path to tackle the question. It is based on the comparison between a given set of papers and carefully chosen sets of papers of the same size, obtained via simulation. This path concentrates on the "quality" of a set of papers of a given size. It should be adapted before it can be used for the comparison of sets of papers of different sizes, as we do here.
} 
the Leiden group (CWTS) and the references cited in Section 6 on this subject). Nonparametric approaches based on percentiles have also been investigated (Bornmann, 2013, Bornmann, Leydesdorff, and Mutz, 2013, Leydesdorff and Bornmann, 2012, Leydesdorff, Bornmann, Mutz, and Opthof, 2011). All approaches relating to this first path require to be able to allocate papers to fields.

The second path was suggested by Zitt and Small (2008) and Zitt (2010, 2011) for ranking journals (see also Pinski and Narin, 1976, Small and Sweeney, 1985, Zitt, Ramanana-Rahary, and Bassecoulard, 2005). It is referred to as "citingside", "a priori", or "source" normalization. Compared with the above approches, its does not require the a priori division of research into fields. The normalization is performed based on the "referencing behavior of citing publications or citing journals" (Waltman and van Eck, 2013a, p. 834). Following Waltman and van Eck (2013a,b), who reformulate proposals often made for journals in terms of publications, it is possible to distinguish three main techniques belonging to this second path.

This first one is similar to the audience factor proposed by Zitt and Small (2008). The citations emitted by a paper have a weight that is inversely proportional to the average number of citations emitted by papers published in the same journal and in the same year.

The second technique in this category consist in the "fractional counting of citations" proposed in Leydesdorff and Opthof (2010a,b) and Glänzel, Schubert, Thijs, and Debackere (2011). For each paper $p$ citing a paper $q$, the "value" of this citation is inversely proportional to the total number of citations made by the paper $p$. Leydesdorff and Opthof (2010a) suggest using all references for the computation of this index. Waltman and van Eck (2013a,b) use only "active" references, i.e., references pointing to a paper in the database within a given timewindow. They argue that, since the coverage of fields in bibliometric database is uneven, considering all references may penalize fields with a low coverage.

The third technique is similar to the Source Normalized Impact per Paper (SNIP) proposed by Moed (2010) and revised in Waltman, van Eck, van Leeuwen, and Visser (2013). The value of a citation emitted by a paper is inversely proportional to the product of the number of active references made by this paper with the proportion of papers published in the same journal and in the same year as this paper having at least one active reference.

This paper is only concerned with fractional counting of citations. It is described in Leydesdorff and Opthof (2010a, p. 2367, col. 2) as "simple and elegant" and in Leydesdorff, Radicchi, Bornmann, Castellano, and de Nooy (2013a, p. 2300, col. 1) as a "radicalized" version of the other techniques. There has been a number of empirical studies on fractional counting of citations. Although some of them give evidence in its favor (Leydesdorff and Shin, 2011, Leydesdorff, Zhou, and Born- 
mann, 2013b, Zhou and Leydesdorff, 2011), others lead to a less positive picture (Bornmann and Marx, 2015, Leydesdorff et al., 2013a, Radicchi and Castellano, 2012a, Ruiz-Castillo, 2014, Waltman and van Eck, 2013a,b).

Our interest in "fractional citation counts" lies in the fact that it is a new way of counting citations that is simple and seems intuitive. A much related idea can also be found in the literature on "domination" in social networks. The " $\beta$ measure" proposed in van den Brink and Gilles $(2000,2002)$ is indeed quite reminiscent of fractional counting with links having a dual interpretation: if a node $p$ dominates a node $q$, this raises the domination score of $p$ by a factor that is inversely proportional to the number of nodes dominating $q$. Moreover, this idea is also at work in PageRank (see Page and Brin, 1998: in this algorithm, the probability that a "random surfer" goes from page $p$ to page $q$ is inversely proportional to the number of outgoing links in page $p$. It should be noted that this idea and, more generally, the underlying algorithm was anticipated by Pinski and Narin, 1976, in the context of ranking journals).

Our aim in this paper is to contribute to a formal study of the properties of fractional counting of citations. This will allow us to analyze the pros and cons of this particular way to count citations from a formal point of view. As in Bouyssou and Marchant (2010), the framework that we use is rich, i.e., much richer ${ }^{2}$ than a framework in which each author is viewed as a collection of papers each having received a certain number of citations as done, e.g., in Bouyssou and Marchant (2011b), Marchant (2009a,b), Quesada (2010), or Woeginger (2008a). We will concentrate on bibliometric rankings instead of bibliometric indices. We study bibliometric rankings of authors instead of bibliometric rankings of journals, as done, e.g., in Bouyssou and Marchant (2011a), Zitt and Small (2008) and Zitt (2010, 2011). We acknowledge the fact that librarians often have to take subscriptions decisions concerning journals coming from different fields. But, comparing authors coming from different fields is also a common practice, e.g., when deciding on promotions ${ }^{3}$.

Since our emphasis is on the formal properties of a particular counting method

\footnotetext{
${ }^{2}$ As pointed out to us by a referee, our framework, although rich, does not include a temporal dimension that would allow us to say that a paper $p$ is written before paper $q$ and that, therefore, $q$ cannot cite $p$.

${ }^{3}$ As pointed out to us by a referee, the question of ranking authors is closely related to the one of ranking research groups (or universities). Indeed, authors will be viewed here as sets of papers. Clearly a similar view can be adopted for research groups. Hence our analysis may also prove useful in this case. Let us however note that the hypothesis made below that all papers have a unique author is less realistic when dealing with research groups. Moreover, in our analysis the ranking of an author will never deteriorate following the publication of a new paper, even if this paper performs poorly in terms of citations. It is not entirely obvious that this hypothesis is also adequate for research groups and that size-independent rankings (in the sense of Waltman and van Eck, 2012b, p. 408) are always adequate in this case.
} 
used as a tool for ranking, there are many important bibliometric problems that we will not consider. For instance, our analysis starts with a given citation graph. Hence, we suppose that we have solved before our analysis the question of the choice of an adequate time window, the choice of the publications to include in this graph (see the problem of trade and profesional magazines in Waltman and van Eck, 2013a, Zitt and Small, 2008). Moreover, when starting with a citation graph, as we do here, all references are supposed to be "active" in the sense of Zitt and Small (2008) and Waltman and van Eck (2013a). Another limitation of this paper is that it does not contain empirical analysis. Hence, we do not deal with the delicate problem of defining what a satisfactory field normalization is (on this point, see Zitt, 2011, Zitt and Cointet, 2014) and how to test it in practice (see Sirtes, 2012 and Waltman and van Eck, 2013a, Appendix C).

The paper is organized as follows. Section 2 presents our setting and introduces the rankings that we will study. Section 3 introduces our conditions on bibliometric ranking functions. Section 4 is devoted to the ranking based on fractional citation counts. Section 5 is devoted to the ranking based on citation counts. Section 6 concludes.

\section{Notation and Setting}

The set of non-negative integers is denoted by $\mathbb{N}$. We define $\mathbb{N}_{+}=\mathbb{N} \backslash\{0\}$. The number of elements of a finite set $\Theta$ will be denoted by $|\Theta|$.

\subsection{Setting}

We use the general framework introduced in Bouyssou and Marchant (2010). We simplify this framework by not taking journals into account. Moreover, in order to keep things simple, we will not deal with co-authors and we will suppose that each paper has a unique author. A proper treatment of co-authors would require using techniques similar to the ones presented in Bouyssou and Marchant (2010). Including this point in this paper would result in much complexity, while trying to solve a question that seems rather independent from the one analyzed here ${ }^{4}$.

The set of authors is denoted by $\mathcal{A}$. It is fixed and finite. We will suppose throughout that $|\mathcal{A}| \geq 3$. The set of all papers is denoted by $\mathcal{P}$. When $a \in \mathcal{A}$ is the author of $p \in \mathcal{P}$, we write $a S p$, i.e., a Signs $p$. Hence, $S$ is a binary relation between the sets $\mathcal{A}$ and $\mathcal{P}$, i.e., a subset of $\mathcal{A} \times \mathcal{P}$. A paper $p \in \mathcal{P}$ may cite other papers in $\mathcal{P}$. When $p \in \mathcal{P}$ cites $q \in \mathcal{P}$, we write $p C q$, i.e., $p$ Cites $q$ (we write

\footnotetext{
${ }^{4}$ However, Waltman and van Eck (2015) have recently argued that the way to take coauthors into account is of importance to evaluate field normalization techniques (see also PerianesRodriguez and Ruiz-Castillo, 2015)
} 
$p \not \subset q$ as an abbreviation for $\operatorname{Not}[p \quad C q])$. Hence, $C$ is a binary relation on $\mathcal{P}$, i.e., a subset of $\mathcal{P} \times \mathcal{P}$. Although we shall suppose throughout that $\mathcal{A}$ is fixed and finite, we shall allow the set of papers $\mathcal{P}$ and, hence, $S$ and $C$ to vary ${ }^{5}$.

A bibliometric profile $G$ on $\mathcal{A}$ is a triple $(\mathcal{P}, S, C)$ with $S \subseteq \mathcal{A} \times \mathcal{P}, C \subseteq \mathcal{P} \times \mathcal{P}$, such that:

- for all $p \in \mathcal{P}$, there is a unique $a \in \mathcal{A}$ such that $a S p$ (i.e., each paper $p$ has a unique author $a$ ),

- there is no cycle ${ }^{6}$ of any length in $C$ (because a paper can only cite older papers).

A bibliometric ranking function (BRF) on $\mathcal{A}$ is a mapping $\succsim$ associating with each bibliometric profile $G$ on $\mathcal{A}$ a weak order $\succsim(G)$ on $\mathcal{A}$. As is usual, we respectively denote by $\succ(G)$ and $\sim(G)$ the asymmetric and symmetric parts ${ }^{7}$ of $\succsim(G)$. The statement $a \succsim(G) b$ (resp. $a \succ(G) b$ and $a \sim(G) b$ ) means that, for the bibliometric profile $G$, author $a$ has a performance that is not worse than (resp. superior to and equivalent to) the performance of $b$.

In order to simplify notation, given a profile $G$, we denote by $P_{G}(a)$ the set of all papers signed by $a \in \mathcal{A}$, i.e.,

$$
P_{G}(a)=\{p \in \mathcal{P}: a S p\} .
$$

Similarly, given a profile $G$, the set of all papers in $\mathcal{P}$ cited by $p \in \mathcal{P}$ is denoted $O C_{G}(p)$ (Qut $\underline{\text { Citations), i.e., }}$

$$
O C_{G}(p)=\{q \in \mathcal{P}: p C q\} .
$$

The set of all papers in $\mathcal{P}$ citing $p \in \mathcal{P}$ is denoted $I C_{G}(p)$ (In $\underline{\text { Citations), i.e., }}$

$$
I C_{G}(p)=\{q \in \mathcal{P}: q C p\} .
$$

Finally, for $p \in \mathcal{P}$, we will sometimes use the notation $S^{-1}(p)$ to denote the author $a \in \mathcal{A}$ such that $a S p$.

\footnotetext{
${ }^{5}$ The way in which the set $\mathcal{P}$ is allowed to vary is not completely formalized here. The simplest way to do so (following what is done in the study of voting rules with a varying population, see, e.g., Young, 1974) would be to consider a countably infinite universe of papers $\mathcal{U}$ that can be identified with $\mathbb{N}$. The admissible sets of papers $\mathcal{P}$ would then be all finite subsets of this universe $\mathcal{U}$.

${ }^{6} \mathrm{~A}$ cycle of length $\ell \geq 1$ in the relation $C$ consists in $\ell$ papers $p^{1}, p^{2}, \ldots, p^{\ell}$ such that $p^{1} C p^{2}$, $p^{2} C p^{3}, \ldots, p^{\ell-1} C p^{\ell}, p^{\ell} C p^{1}$.

${ }^{7} \mathrm{~A}$ weak order on a set is a complete $(a \succsim b$ or $b \succsim a$, for all $a, b)$ and transitive binary relation $(a \succsim b$ and $b \succsim c$ imply $a \succsim c$, for all $a, b, c)$ on this set. The asymmetric part (resp. symmetric

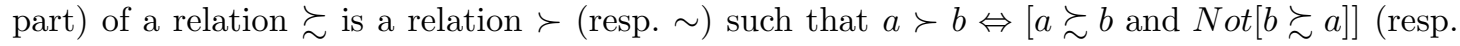
$a \sim b \Leftrightarrow[a \succsim b$ and $b \succsim a])$.
} 


\section{Remark 1}

Our setting has relations with the classical problem of ranking objects on the basis of a binary relation between these objects (see, e.g., Bouyssou, 2004, Dutta and Laslier, 1999, Laslier, 1997, Rubinstein, 1980, Schwartz, 1972). There are however important differences that prevent us from simply recycling known results. We want to compare authors but a bibliometric profile does not give a binary relation on the set of authors. In our setting, authors are compared on the basis of a binary relation (the citing relation) on the set of papers. Another relation (the signing relation) links papers and authors. Moreover, the citing relation which is the basis of our analysis is supposed to be without any cycle. This sharply contrasts with the setting of the papers mentioned above. The relation on the set of objects that they consider is usually complete and may have cycles, e.g., being a tournament on this set (i.e., a complete and antisymmetric binary relation, in view of the classical result of McGarvey, 1953). The citing relation is without any cycle and is obviously incomplete (see however Kóczy and Strobel, 2007, for the use of techniques coming from the literature on tournaments for the ranking of journals).

The papers that come closer to our analysis are the papers devoted to the study of the $\beta$ domination measure (van den Brink and Gilles, 2000, 2002). However, these papers do not suppose that the underlying graph is without any cycle and do not involve a notion of "author". Moreover, they study the index given by the $\beta$ domination measure and not the ranking that it induces. Similar remarks apply to the papers devoted to the study of the ranking that PageRank induces on the set of pages from an axiomatic point of view (Altman, 2005, Altman and Tennenholtz, 2005). Notice that in our setting the citation graph that is used is not valued. This contrasts with the setting used by Palacios-Huerta and Volij (2004) and Demange (2014) for the study of iterative ranking procedures for journals. In these papers a link between journals $j$ and $j^{\prime}$ carries a valuation that is interpreted as the number of citations from $j$ to $j^{\prime}$.

\section{Remark 2}

Our proofs will often lead us to consider changes in the citation relation. We leave to the reader the, easy, task of checking that the changes that we will consider will never create cycles in this relation. We will not stress this point in what follows.

We are fully aware that the hypothesis that the graph we use is without any cycle is not always satisfied: in practice, papers being elaborated simultaneously can cite each other. We nevertheless maintain it because we feel that it is a crucial feature of citations graphs when compared to other "social network" graphs. Moreover, in practice, cycles in the citing relation only involve a very small percentage of all papers. Most of our analysis does not crucially depend on this hypothesis. At the cost of minor modifications of our axioms, all results presented below can be extended to cover the case of citation graphs having cycles. 


\subsection{Definitions}

\subsubsection{Ranking based on citations}

We may want to rank authors based on the number of citations that their papers receive. This is a standard bibliometric index (see, e.g., van Raan, 2006). It has been studied from an axiomatic point of view by Marchant (2009a) and Bouyssou and Marchant (2014), using a setting that is different from the one used here. This leads to associating with each profile $G=(\mathcal{P}, S, C)$ on $\mathcal{A}$ and with each author $a \in \mathcal{A}$ the index:

$$
f_{c}(a, G)=\sum_{p \in P_{G}(a)}\left|I C_{G}(p)\right|
$$

The index $f_{c}$ records for each paper $p$ signed by author $a$ (i.e., $\left.p \in P_{G}(a)\right)$ the number of citations received by $p$ (i.e., the cardinality of the set $\left.I C_{G}(p)\right)$. These numbers are then added to give the score of author $a$ for the bibliometric profile $G$, i.e., $f_{c}(a, G)$. Authors are then ranked according to the values of $f_{c}$, i.e., for all profiles $G$ on $\mathcal{A}$ and all $a, b \in \mathcal{A}$,

$$
a \succsim_{c}(G) b \Leftrightarrow f_{c}(a, G) \geq f_{c}(b, G) .
$$

As discussed above, a weakness of this way to rank authors is that it does not take the specific characteristics of "fields" into account.

\subsubsection{Ranking based on normalized citations}

This ranking involves a "local" normalization of citations: the field of a paper $p$ is the set of all papers citing it. For each paper $q$ citing a paper $p$, the "value" of this citation is inversely proportional to the total number of citations made by the paper $q$ (see Glänzel et al., 2011, Leydesdorff and Opthof, 2010a). Formally, this leads to associating with each profile $G=(\mathcal{P}, S, C)$ on $\mathcal{A}$ and with each author $a \in \mathcal{A}$ the index:

$$
f_{n}(a, G)=\sum_{p \in P_{G}(a)}\left[\sum_{q \in I C_{G}(p)} \frac{1}{\left|O C_{G}(q)\right|}\right]
$$

(we use the convention that summing over an empty index set gives the value $0)$. The index $f_{n}$ works as follows. For each paper $p$ authored by author $a$ (i.e., $p \in P_{G}(a)$ ), we consider the set of papers $q$ citing $p$ (i.e., the papers $q \in I C_{G}(p)$ ). If the paper $q$ citing $p$ gives $k$ citations, the index considers that the value of the citation from $q$ to $p$ is $1 / k$. These values are computed for all papers citing $p$ and are then added to give the contribution of paper $p$ to the standing of its author. Doing so for each paper $p$ authored by $a$, we finally add up these numbers to 
compute the score of author $a$ in the profile $G$, i.e., $f_{n}(a, G)$. Authors are then ranked according to the values of $f_{n}$, i.e., for all profiles $G$ on $\mathcal{A}$ and all $a, b \in \mathcal{A}$,

$$
a \succsim_{n}(G) b \Leftrightarrow f_{n}(a, G) \geq f_{n}(b, G) .
$$

The aim of this paper is to give conditions characterizing the BRF $\succsim_{n}$. We will do the same for $\succsim_{c}$ so as to have a point of comparison. Although there are other characterizations of $\succsim_{c}$ (see Bouyssou and Marchant, 2010) and Bouyssou and Marchant (2014), Marchant (2009a), these works use settings that are different from the one used here (see Section 6). The one used in Bouyssou and Marchant (2014), Marchant (2009a) is simpler than the one used here and does not allow to tackle the question of field normalization. The one used in Bouyssou and Marchant (2010) is more complex since it also involves journals and co-authors. This added complexity implies using more involved conditions.

\section{Conditions on bibliometric ranking functions}

This section introduces and discusses a number of conditions that a BRF may possess. The first three of them are classical.

Our first condition is author anonymity. It roughly says that the way in which authors are labelled does not influence the ranking. In particular, if two authors $a$ and $b$ exchange their papers, everything else being kept unchanged, they should exchange their positions in the ranking. This condition is clearly satisfied by $\succsim_{n}$. Indeed, starting with the initial profile $G$, let us define the profile $H$ by exchanging the papers signed by $a$ and $b$. It is clear that we have $f_{n}(a, G)=f_{n}(b, H)$ and $f_{n}(b, G)=f_{n}(a, H)$, while, for all $c \in \mathcal{A} \backslash\{a, b\}, f_{n}(c, G)=f_{n}(c, H)$.

A1 (Author Anonymity) Let $G=(\mathcal{P}, S, C)$ and $a, b \in \mathcal{A}$. Consider now the profile $H=\left(\mathcal{P}, S^{*}, C\right)$ that is identical to $G$, except that $P_{G}(a)=P_{H}(b)$ and $P_{G}(b)=P_{H}(a)$. Then, we have $a \succsim(G) b \Leftrightarrow b \succsim(H) a$.

Consider a profile $G$ and two authors $a$ and $b$. Suppose that a BRF leads to declare that $a$ is not worse than $b$ (resp. $a$ better than $b$ ). Let us now consider a profile that is identical to $G$ except that a paper signed by $a$ has received an additional citation (we suppose that this addition citation does not create cycles in the citation relation). In this new profile $G^{*}$, using the same BRF as before, we expect that $a$ remains not worse than $b$ (resp. better than $b$ ). It is easy to check that this is the case with both $\succsim_{n}$ and $\succsim_{c}$. This property is a classical monotonicity condition. We shall need a stronger version of this condition. In $G^{*}$, we would like $a$ to be ranked strictly above $b$. This is the case with $\succsim_{c}$, as can easily be checked. But this fails with $\succsim_{n}$. If the citation that is added to one paper 
signed by $a$ is coming from a paper that previously only cited papers signed by $a$, it is clear that, with $f_{n}$, the score of $a$ does not change. In order to ensure that $a$ is now strictly above $b$, we have to impose a constraint on the papers originally cited by the paper giving the additional citation. This condition is called strong monotonicity. It is the only one of our conditions that will delineate cases in which an author is ranked strictly above another one.

A2 (Strong Monotonicity) Let $G=(\mathcal{P}, S, C)$ and $a, b \in \mathcal{A}$. Let $p, q \in \mathcal{P}$ such that $q \in P_{G}(a), p \Phi^{*} q$ and $q \varnothing^{*} p$. Let $H=\left(\mathcal{P}, S, C^{*}\right)$ be a profile such that $C^{*} \backslash C=\{(p, q)\}$. If $a \succsim(G) b$ (resp. $\left.a \succ(G) b\right)$ then $a \succsim(H) b$ (resp. $\left.a \succ(H) b\right)$.

Moreover, if $a \succsim(G) b$ and either $O C_{G}(p)=\varnothing$ or there is $r \in O C_{G}(p)$ such that $r \notin P_{G}(a)$, then $a \succ(H) b$.

Strong monotonicity is satisfied by $\succsim_{n}$. Indeed, if, starting with the profile $G$, we define the profile $H$ by the addition of a citation to a paper signed by $a$, we have: $f_{n}(a, H) \geq f_{n}(a, G)$, while, for all $c \in \mathcal{A} \backslash\{a\}, f_{n}(c, H) \leq f_{n}(c, G)$. The inequality $f_{n}(a, H) \geq f_{n}(a, G)$ becomes strict if we impose that the additional citation comes from a paper that, previously, did not cite only papers signed by $a$.

Our next condition deals with the fact that we want to rank authors on the basis of the citations received by the papers they sign and not on the basis of the number of papers they sign. It says that if two profiles $G$ and $H$ only differ by the addition of a paper that does not cite any other paper and is not cited by any paper, we will call such papers dummy papers, then the ranking of all authors should remain the same. Among all our conditions, it is the only one that involves changes to the set of papers in the profile. This condition is clearly satisfied by $\succsim_{n}$. Indeed if, starting with a profile $G$, we obtain the profile $H$ by the addition of a dummy paper, we have $f_{n}(c, H)=f_{n}(c, G)$, for all $c \in \mathcal{A}$.

A3 (Dummy Paper) Let $G=(\mathcal{P}, S, C), a \in \mathcal{A}$, and $v \notin \mathcal{P}$. Consider now the profile $H=\left(\mathcal{P}^{*}, S^{*}, C\right)$ with $\mathcal{P}^{*}=\mathcal{P} \cup\{v\}$ and $S^{*}=S \cup\{(a, v)\}$. Then, we have $\succsim(G)=\succsim(H)$.

The next three conditions are slightly more complex.

Consider two papers $r$ and $s$. Suppose that these two papers do not receive any citation. Suppose furthermore that each of them cites $k$ papers. Intuitively, it seems that the value of the citations emitted by $r$ and $s$ are quite similar: both are uncited and cite the same number of papers. Our next condition formalizes this similarity. It says that if two papers $r$ and $s$ are uncited and if both cite $k \in \mathbb{N}_{+}$ papers, then, if the paper $p$, signed by $a$, is cited by $r$ and does not cite any paper, and if the paper $q$, signed by $b$, is cited by $s$ and does not cite any paper, the two citations (from $r$ to $p$ and from $s$ to $q$ ) can be exchanged without altering the position of $a$ with respect to (henceforth, w.r.t.) $b$ in the ranking. Said differently, 
all citations coming from uncited papers citing the same number of papers have the same impact. This condition is clearly satisfied by $\succsim_{n}$. Indeed if, starting with a profile $G$, we obtain the profile $H$ by exchanging citations coming from uncited papers giving the same number of citations, we have $f_{n}(c, H)=f_{n}(c, G)$, for all $c \in \mathcal{A}$. The name of this condition has been chosen so as to avoid any confusion with the condition called Source Independence used in Bouyssou and Marchant (2010, p. 362).

A4 (Restricted Source Independence) Let $G=(\mathcal{P}, S, C)$, and $p, q, r, s \in \mathcal{P}$ be such that $I C_{G}(r)=I C_{G}(s)=\varnothing,\left|O C_{G}(r)\right|=\left|O C_{G}(s)\right|, r C p, s C q, r \phi q$, $s \ell^{\prime} p, O C_{G}(p)=O C_{G}(q)=\varnothing$. Consider now the profile $H=\left(\mathcal{P}, S, C^{*}\right)$ that is identical to $G$, except that $r{\phi^{*}}^{*}, s{C^{*}}^{*}, r C^{*} q$, and $s C^{*} p$. Then, we have $\succsim(G)=\succsim(H)$.

The next condition says that if a paper is uncited and does not cite any paper signed by authors $a$ and $b$, we can delete a citation from this paper to any paper it cites, without altering the relative position of authors $a$ and $b$ in the ranking. This condition is clearly satisfied by $\succsim_{n}$. Indeed, suppose that the profile $G$ is such that there is a paper $p$ that does not cite any paper signed by $a$ or by $b$. Deleting a citation from the paper $p$ to any paper that is not signed by $a$ or by $b$ defines the profile $H$. It is easy to check that we have $f_{n}(a, H)=f_{n}(a, G)$ and $f_{n}(b, H)=f_{n}(b, G)$.

A5 (Independence of Irrelevant Citations) Let $G=(\mathcal{P}, S, C)$ and $a, b \in \mathcal{A}$. Let $p \in \mathcal{P}$ be such that $I C_{G}(p)=\varnothing$ and, for all $r \in O C_{G}(p), a \$ p$ and $b \$ p r$. Let $q \in O C_{G}(p)$. Consider now the profile $H=\left(\mathcal{P}, S, C^{*}\right)$ that is identical to $G$ except that $p{C^{*}}^{*}$. Then, we have $a \succsim(G) b \Leftrightarrow a \succsim(H) b$.

The above condition would be violated by a BRF in which a local modification of the citation graph may have consequences that are not only "local", e.g., if the performance of the author of a paper influences the value of a citation made by this paper.

It is not difficult to check that all conditions introduced so far are satisfied not only by $\succsim_{n}$ but also by $\succsim_{c}$. Hence, in order to characterize $\succsim_{n}$, we need conditions that are satisfied by $\succsim_{n}$ but are violated by $\succsim_{c}$. It turns out that only one additional condition will be needed.

Consider a profile $G$ in which an uncited paper $p$ cites $\ell$ papers $q^{1}, q^{2}, \ldots, q^{\ell}$. For each $q^{j} \in O C_{G}(p)$, let us suppose that there are $k \in \mathbb{N}_{+}$papers $q_{1}^{j}, q_{2}^{j}, \ldots, q_{k}^{j}$, all signed by the same author as $q^{j}$ and that are not cited by $p$. Consider now the profile $H$ that is identical to $G$, except that, for all $j \in\{1,2, \ldots, \ell\}$ and for all $i \in\{1,2, \ldots, k\}$, we have added a citation from $p$ to $q_{i}^{j}$. It is clear that such a modification can only affect the values of $f_{n}$ for authors signing papers that 
were initially cited by $p$. But, it is easy to see that this modification does not affect these values. Indeed, supposing that $k=1$, the value of each of the original citations has been divided by 2 , while each of them has been duplicated. Hence, we have, for all $a \in \mathcal{A}, f_{n}(a, H)=f_{n}(a, G)$.

A6 (Citation Intensity Independence) Let $G=(\mathcal{P}, S, C)$ and $p \in \mathcal{P}$. Let $k \in \mathbb{N}_{+}$. Suppose that $I C_{G}(p)=\varnothing$ and $O C_{G}(p)=\left\{q^{1}, q^{2}, \ldots, q^{\ell}\right\}$. Suppose furthermore that, for each $j \in\{1,2, \ldots, \ell\}$, there are $q_{1}^{j}, q_{2}^{j}, \ldots, q_{k}^{j} \in \mathcal{P}$ that are all signed by the same author as $q^{j}$ and that are not cited by $p$. Consider now the profile $H=\left(\mathcal{P}, S, C^{*}\right)$ that is identical to $G$ except that, for all $j \in\{1,2, \ldots, \ell\}$ and all $i \in\{1,2, \ldots, k\}$, we have added a citation from $p$ to $q_{i}^{j}$. Then, we have $\succsim(G)=$ $\succsim(H)$.

\section{Characterization of $\succsim_{n}$}

\subsection{Result}

The conditions introduced so far allow us to characterize the BRF $\succsim_{n}$.

\section{Theorem 1}

$A$ BRF $\succsim$ on $\mathcal{A}$ satisfies conditions $A 1-A 6$ iff $\succsim=\succsim_{n}$.

The necessity of the six conditions was discussed above. The proof of sufficiency is given in the next subsection. We will also show that the conditions used above are independent: omitting any one of them invalidates the result.

\subsection{Sufficiency proof of Theorem 1}

Let us start with a simple lemma showing that, without altering the ranking, a citation emitted by an uncited paper to a paper $p$ can be transferred to another paper $q$ signed by the same author as $p$.

\section{Lemma 1}

Suppose that a BRF $\succsim$ satisfies A3 (Dummy Paper) and A6 (Citation Intensity Independence). Let $G=(\mathcal{P}, S, C)$. Let $r \in \mathcal{P}$ such that $I C_{G}(r)=\varnothing$. Let $H=$ $\left(\mathcal{P}, S, C^{*}\right)$ be a profile that is identical to $G$, except that, for some $a \in \mathcal{A}$ and some $p, q \in \mathcal{P}$, we have: $a S p$ and $a S q, r C p$ and $r \not q, r C^{*} q$ and $r \mathbb{C}^{*} p$. Then, we have $\succsim(G)=\succsim(H)$.

\section{ProOF}

Let $r \in \mathcal{P}$ be such that $I C_{G}(r)=\varnothing$. Consider the set of all papers cited by $r \in \mathcal{P}$, i.e., $O C_{G}(r)$. By construction, we know that $p \in O C_{G}(r)$ and $q \notin O C_{G}(r)$. Suppose that $O C_{G}(r)=\left\{p, t^{1}, t^{2}, \ldots, t^{\ell}\right\}$ with $\ell \in \mathbb{N}$. 
Using A3 (Dummy Paper), we duplicate all papers in $O C_{G}(r) \backslash\{p\}$, to create the papers $t^{* 1}, t^{* 2}, \ldots, t^{* \ell}\left(t^{* j}\right.$ is signed by the same author as $t^{j}$. It does not give or receive citations). For this new profile $G_{1}$, we have $\succsim(G)=\succsim\left(G_{1}\right)$.

We then use A6 (Citation Intensity Independence) applied with $k=1$ to the paper $r$. In the profile $G_{2}$ that we obtain, $r$ cites $p, q$, and, for all $j \in\{1,2, \ldots, \ell\}$, $t^{j}$ and $t^{* j}$. For this new profile $G_{2}$, we have $\succsim\left(G_{1}\right)=\succsim\left(G_{2}\right)$.

Starting from $G_{2}$, consider now the profile $G_{3}$ in which the citation from $r$ to $p$ has been suppressed as well as all citations from $r$ to the papers $t^{* j}$. Using A6 (Citation Intensity Independence), we obtain $\succsim\left(G_{3}\right)=\succsim\left(G_{2}\right)$.

In the profile $G_{3}$, the papers $t^{* j}$ are dummy papers. We can suppress them, using A3 (Dummy Paper). This leads to the profile $G_{4}$ and we know that $\succsim\left(G_{4}\right)=$ $\succsim\left(G_{3}\right)$. It is easy to see that $G_{4}=H$, which completes the proof.

\section{Proof of Theorem 1}

Let us consider a bibliometric profile $G=(\mathcal{P}, S, C)$ on $\mathcal{A}$. Take $a, b \in \mathcal{A}$ with $a \neq b$. Because we have supposed that $|\mathcal{A}| \geq 3$, let us choose $c \in \mathcal{A} \backslash\{a, b\}$.

Step 1. We build a profile $G_{1}$ such that $\succsim$ ranks all authors as in $G$ and in which a paper either cites other papers or is cited by other papers but not both.

Because we have supposed that the citation graph is without any cycle, there is a paper $p \in \mathcal{P}$ such that $I C_{G}(p)=\varnothing$.

If $O C_{G}(p)=\varnothing$, paper $p$ is a dummy paper. Using A3 (Dummy Paper), we can suppress it from $\mathcal{P}$.

Otherwise, using A3 (Dummy Paper), we duplicate all the papers in $O C_{G}(p)$ (a duplicate is signed by the same author as the duplicated paper. A duplicate does not cite any paper and is not cited by any paper). Using Lemma 1, we transfer each citation from $p$ to the papers in $O C_{G}(p)$ to the duplicated papers. The paper $p$ that was uncited in $G$ is said to have been processed.

In either case, the new citation graph that we have built is still without any cycle. Hence there must exist an unprocessed paper from the original set of papers that is uncited. We then repeat the above operations. We do so until we have processed all papers in the original set of papers. This defines the profile $G_{1}$. The ranking of $a$ w.r.t. $b$ in $G_{1}$ is the same as in $G$.

Notice that in $G_{1}$, a paper is either a sender (cites other papers but is not cited) or a receiver (is cited by other papers but does not cite any paper). By construction, all receivers are cited by a unique sender.

Step 2. We build a profile $G_{2}$ in which all senders are signed by $c$.

Consider a sender $p$ in $G_{1}$ that is not signed by $c$. Using A3 (Dummy Paper), we duplicate $p$ as well as all papers in $O C_{G_{1}}(p)$ (all these duplicates are signed by $c)$. Using A5 (Independence of Irrelevant Citations), we can add a citation from the duplicate of $p$ to the duplicate of papers in $O C_{G_{1}}(p)$. Using A4 (Restricted 
Source Independence), we can exchange the citations emitted by $p$ and the citations emitted by its duplicate. Using A5 (Independence of Irrelevant Citations), we can suppress all citations emanating from $p$. The duplicate of the papers in $O C_{G_{1}}(p)$ as well as $p$ are now dummy papers. We can suppress them, using A3 (Dummy Paper).

Repeating the above operations for all senders in $G_{1}$ defines the profile $G_{2}$. The ranking of $a$ w.r.t. $b$ in $G_{2}$ is the same as in $G_{1}$.

Step 3. We build a profile $G_{3}$ in which all senders cite the same number of papers.

In $G_{2}$, we compute the number of citations given by each sender. Let $K$ be the least common multiple (henceforth, l.c.m.) of all these numbers (any common multiple could be used). We use A3 (Dummy Paper) to create dummy papers signed by $c$. We then use A6 (Citation Intensity Independence) to transform this profile into a profile in which all senders cite exactly $K$ papers. Note that, since we may create as many dummy papers signed by $c$ as we wish, we can do this in such a way that each receiver is cited by exactly one sender. This creates a new profile $G_{3}$ in which the ranking of $a$ w.r.t. $b$ is the same as in $G_{2}$.

Step 4. We build a profile $G_{4}$ in which all receivers are signed by $a, b$, or $c$.

Consider a receiver in $G_{3}$ that is signed by an author that does not belong to $\{a, b, c\}$. We use A3 (Dummy Paper) to create $K+1$ dummy papers all signed by $c$. Using A5 (Independence of Irrelevant Citations), we add citations from one of these new papers to the $K$ other new papers. Using A4 (Restricted Source Independence), we can exchange the citation to the receiver not signed by $a, b$ or $c$ that we are processing with a citation to one of the new receivers signed by $c$. Once this is done, we use A5 (Independence of Irrelevant Citations) to suppress the citations from the new sender signed by $c$ to the $K-1$ receivers signed by $c$ and to the receiver not signed by $a, b$ or $c$ that we are processing. After this is done, all these papers become dummy papers and we can suppress them, using A3 (Dummy Paper). All these operations do not alter the ranking of $a$ w.r.t. $b$. They lead to a decrease in the number of receivers not signed by $a, b$ or $c$ by one unit. We then repeat these operations until all receivers are signed by $a, b$ or $c$.

This defines the profile $G_{4}$. In this profile, all senders are signed by $c$. They all cite exactly $K$ receivers. All receivers are signed by $a, b$ or $c$. They all receive exactly one citation. In $G_{4}$, the ranking of $a$ w.r.t. $b$ is the same as in $G_{3}$.

Step 5. We build a profile $G_{5}$ in which each sender cites at least $K-1$ papers signed by $c$.

In $G_{4}$, there may exist senders citing papers signed by $a$ and at the same time papers signed by $b$ or several papers signed by $a$ or $b$. Using A3 (Dummy Paper) and A5 (Independence of Irrelevant Citations), we can add to this profile as many 
senders signed by $c$ citing $K$ receivers signed by $c$ as we wish. We can therefore use A4 (Restricted Source Independence) to obtain a profile in which each sender cites: $(i)$ one paper signed by $a$ and $K-1$ papers signed by $c$, or (ii) one paper signed by $b$ and $K-1$ papers signed by $c$, or $(i i i) K$ papers signed by $c$.

This defines the profile $G_{5}$. In $G_{5}$, the ranking of $a$ w.r.t. $b$ is the same as in $G_{4}$.

Suppose now that, in the initial profile $G$, we had $f_{n}(a, G)=f_{n}(b, G)$. Let us show that we must have $a \sim(G) b$.

It is easy to see that, in the profile $G_{5}$, the total number of citations received by papers signed by $a$ is equal to the total number of citations received by papers signed by $b$.

Step 6. With $G_{5}$, we have a profile that is symmetric w.r.t. $a$ and $b$. There are a number of senders signed by $c$ citing only one receiver signed by $a$ and $K-1$ receivers signed by $c$. There are exactly the same number of senders citing only one paper signed by $b$ and $K-1$ receivers signed by $c$. All other senders are signed by $c$ and cite $K$ receivers signed by $c$.

To each sender, signed by $c$, citing one receiver signed by $a$ and $K-1$ receivers signed by $c$, we can associate a sender, signed by $c$, citing one receiver signed by $b$ and $K-1$ receivers signed by $c$. These two senders have different names, as well as all the papers that they cite. Using A3 (Dummy Paper), A4 (Restricted Source Independence), and A5 (Independence of Irrelevant Citations), the name of the senders and the receivers is immaterial. Using A1 (Author Anonymity), the identity of the authors $a$ and $b$ is irrelevant for ranking them. Hence, they must be ranked equally.

Let us detail this step of reasoning. Consider a sender $p$ citing $K-1$ receivers signed by $c$ and the receiver $q$ signed by $a$. Consider furthermore a sender $p^{*}$ citing $K-1$ receivers signed by $c$ and the receiver $q^{*}$ signed by $b$.

Let us show that in the profile in which everything is unchanged, except that $q$ is signed by $b$ and $q^{*}$ is signed by $a$, the ranking of $a$ w.r.t. $b$ is unchanged. This is done as follows. Using A3 (Dummy Paper), we create a paper $v$ signed by $a$ and a paper $v^{*}$ signed by $b$. Using Lemma 1 , we can transfer the citation from $p$ to $q$ to $v$ and the citation from $p^{*}$ to $q^{*}$ to $v^{*}$. In this new profile, the papers $q$ and $q^{*}$ do not send or receive citations. Using A3 (Dummy Paper), they can be suppressed from the profile. Using A3 (Dummy Paper) again, we create the paper $q^{*}$ signed by $a$ and the paper $q$ signed by $b$. Using Lemma 1, we can transfer the citation from $p$ to $v$ to $q^{*}$ and the citation from $p^{*}$ to $v^{*}$ to $q$. After these transfers, the papers $v$ and $v^{*}$ do not send or receive citations. Using A3 (Dummy Paper), we can therefore suppress them from the profile. Hence, we obtain a profile in which $p$ cites $K-1$ receivers signed by $c$ and the receiver $q^{*}$ signed by $a$ and $p^{*}$ cites $K-1$ 
receivers signed by $c$ and the receiver $q$ signed by $b$. The ranking of $a$ w.r.t. $b$ in this new profile is the same as in the original one. Since $p$ and $p^{*}$ are uncited and both cite $K$ papers, we can use A4 (Restricted Source Independence) to exchange the citation from $p$ to $q^{*}$ and the citation from $p^{*}$ to $q$. The ranking of $a$ w.r.t. $b$ in this new profile is the same as in the original one.

The above operations can be applied to all pairs of senders, the first one citing one paper signed by $a$ and the second one citing one paper signed by $b$. Once these operations have been applied, we use A1 (Author Anonymity) to exchange the papers signed by the authors $a$ and $b$. This exchanges the position of $a$ and $b$ in the ranking. This leads to the profile from which we started at the beginning of Step 7 . Hence, in the initial profile, $a$ and $b$ must belong to the same equivalence class.

This shows that $a$ and $b$ must be ranked equally in $G_{5}$. Hence, they must also be ranked equally in $G$, i.e., $a \sim(G) b$.

Suppose now that in the initial profile $G$ we had $f_{n}(a, G)>f_{n}(b, G)$. Let us show that we must have $a \succ(G) b$, which will complete the proof.

It is easy to see that, in $G_{5}$, the number of citations to papers signed by $a$ is greater than the number of citations to papers signed by $b$.

Step 7. Consider the profile $G_{5}$. Let $x$ be the number of citations to papers signed by $a$ minus the number of citations to papers signed by $b$ in this profile.

We can decrease the number of citations to papers by $a$ in $G_{5}$, without changing its structure, so that the papers signed by $a$ and $b$ receive the same total number of citations. More precisely, this is done as follows. We first create, using A3 (Dummy Paper), $x$ new receivers all signed by $c$. We then replace $x$ citations to papers signed by $a$ by citations to these new receivers. This creates $x$ dummy papers signed by $a$.

We know that, in this modified profile, $a$ and $b$ are equally ranked.

Take one of the $x$ dummy papers signed by $a$ that we have just created. Let us call it $p$. We have previously replaced the citation from a sender, called $s$ and signed by $c$, to $p$ by a citation from $s$ to a new receiver signed by $c$, called $v$. In this profile we know that $a$ and $b$ belong to the same equivalence class. Using A5 (Independence of Irrelevant Citations), we can suppress the citation from $s$ to $v$, without affecting the position of $a$ w.r.t. $b$ in the ranking. The paper $v$ signed by $c$ is now a dummy paper. We can suppress it, thanks to A3 (Dummy Paper), without altering the ranking of $a$ w.r.t. $b$. We now add a citation from $s$ to $p$. Using A2 (Strong Monotonicity), we must have $a$ strictly above $b$ in this profile.

Repeating this process for the $x$ dummy papers signed by $a$ created at the beginning of this step, shows that in $G_{5}$ and, hence, in $G, a$ must be ranked strictly above $b$. 
This completes the proof.

\subsection{Independence of the conditions in Theorem 1}

We give here examples showing that the conditions used in Theorem 1 are independent.

Example 1 (A1 (Author Anonymity))

Choose $z \in \mathcal{A}$. Consider the BRF such that, for all bibliometric profiles $G$ on $\mathcal{A}$, and all $a, b \in \mathcal{A}$,

$$
a \succsim(G) b \Leftrightarrow g(a, G) \geq g(b, G)
$$

with

$$
g(a, G)=\left\{\begin{aligned}
f_{n}(a, G) & \text { if } a \neq z \\
2 f_{n}(a, G) & \text { if } a=z .
\end{aligned}\right.
$$

Condition A1 (Author Anonymity) is violated. It is easy to check that all other conditions hold.

Example 2 (A2 (Strong Monotonicity))

It is easy to check that the BRF always returning a weak order having one equivalence class violates A2 (Strong Monotonicity). All other conditions trivially hold. $\diamond$

Example 3 (A3 (Dummy Paper))

Consider the BRF such that, for all bibliometric profiles $G$ on $\mathcal{A}$, and all $a, b \in \mathcal{A}$,

$$
a \succsim(G) b \Leftrightarrow g(a, G) \geq g(b, G)
$$

with

$$
g(a, G)=f_{n}(a, G)+\left|P_{G}(a)\right| .
$$

The BRF is based on an index that adds up the score $f_{n}(a, G)$ with the number of papers $\left|P_{G}(a)\right|$ of each author $a \in \mathcal{A}$. It is clear that this BRF violates A3 (Dummy Paper). It is simple to check that all other conditions hold.

Example 4 (A4 (Restricted Source Independence))

Consider the BRF such that, for all bibliometric profiles $G$ on $\mathcal{A}$, and all $a, b \in \mathcal{A}$,

$$
a \succsim(G) b \Leftrightarrow g(a, G) \geq g(b, G)
$$

with

$$
g(a, G)=\sum_{p \in P_{G}(a)}\left[\sum_{q \in I C_{G}^{\prime}(p)} \frac{1}{\left|O C_{G}(q)\right|}+\sum_{q \in I C_{G}^{\prime \prime}(p)} \frac{1}{2\left|O C_{G}(q)\right|}\right]
$$


where

$$
\begin{aligned}
& I C_{G}^{\prime}(p)=\left\{q \in \mathcal{P}: q C p \text { and } S^{-1}(q) \neq S^{-1}(p)\right\}, \\
& I C_{G}^{\prime \prime}(p)=\left\{q \in \mathcal{P}: q C p \text { and } S^{-1}(q)=S^{-1}(p)\right\} .
\end{aligned}
$$

This BRF is close to $\succsim_{n}$ but treats self-citations differently from other citations. It is clear that this BRF violates A4 (Restricted Source Independence).

For instance, let $\mathcal{A}=\{a, b, c\}$ and $G=(\mathcal{P}, S, C)$ a bibliometric profile on $\mathcal{A}$ with $\mathcal{P}=\left\{p, q, r, p^{*}, q^{*}, r^{*}\right\}$. Suppose that the signing relation $S$ is such that: $P_{G}(a)=\left\{p, q^{*}, r^{*}\right\}, P_{G}(b)=\left\{p^{*}\right\}$, and $P_{G}(c)=\{q, r\}$. Suppose finally that the citing relation $C$ is such that: $q C p, r C p, q^{*} C p^{*}, r^{*} C p^{*}$. It is easy to check that we have $a \sim(G) b$.

Consider now the profile $H=\left(\mathcal{P}, S, C^{*}\right)$ that is identical to $G$, except that the citation relation is now such that: $q^{*} C^{*} p, r C^{*} p, q C^{*} p^{*}, r^{*} C^{*} p^{*}$. It is easy to check that we have $b \succ(G) a$, violating A4 (Restricted Source Independence).

All other conditions trivially hold.

Example 5 (A5 (Independence of Irrelevant Citations))

Given a profile $G=(\mathcal{P}, S, C)$. Let us consider a real-valued function $h(p, G)$ such that $h(p, G)=0$ if $O C_{G}(p)=\varnothing$ and $h(p, G)=\epsilon$ if $O C_{G}(p) \neq \varnothing$ with $\epsilon<1 /|\mathcal{P}|$. $\mathcal{A}$,

Consider the BRF such that, for all bibliometric profiles $G$ on $\mathcal{A}$, and all $a, b \in$

$$
a \succsim(G) b \Leftrightarrow g(a, G) \geq g(b, G)
$$

with

$$
g(a, G)=\sum_{p \in P_{G}(a)}\left(\left[\sum_{q \in I C_{G}(p)} \frac{1}{\left|O C_{G}(q)\right|}\right]-h(p, G)\right) .
$$

This BRF violates A5 (Independence of Irrelevant Citations). For instance, let $\mathcal{A}=\{a, b, c\}$ and $G=(\mathcal{P}, S, C)$ a bibliometric profile on $\mathcal{A}$ such that $\mathcal{P}=$ $\left\{\alpha^{1}, \alpha^{2}, \beta^{1}, \beta^{2}, \gamma, p, q\right\}$.

Suppose that the signing relation $S$ is such that:

$$
\begin{aligned}
& P_{G}(a)=\{p\}, \\
& P_{G}(b)=\{q\}, \\
& P_{G}(c)=\left\{\alpha^{1}, \alpha^{2}, \beta^{1}, \beta^{2}, \gamma\right\} .
\end{aligned}
$$

Suppose finally that the citing relation $C$ is such that:

$$
\begin{aligned}
& \alpha^{1} C p, \alpha^{2} C p, \\
& \beta^{1} C q, \beta^{2} C q .
\end{aligned}
$$

It is easy to check that we have $a \sim(G) b$. If we define the profile $H=\left(\mathcal{P}, S, C^{*}\right)$ in which $C^{*}$ is identical to $C$, except that we have added a citation from $p$ to $\gamma$ to $G$, we obtain $b \succ(H) a$. 
It is not difficult to check that all other conditions are satisfied. For instance, A6 (Citation Intensity Independence) is satisfied because citations that are added in this condition are sent by papers that already cite other papers. Hence, for these papers, the function $h(p, G)$ does not change. Because the function $h(p, G)$ is always strictly less that $1 /|\mathcal{P}|$, the addition of a citation from a paper signed by $a$ to another paper signed by $a$ always improves $g(a, G)$. Hence, A2 (Strong Monotonicity) is always satisfied. A4 (Restricted Source Independence) is satisfied because this condition leaves unchanged the number of citations given and received by all papers.

Example 6 (A6 (Citation Intensity Independence))

It is easy to check that the $\mathrm{BRF} \succsim_{c}$ violates A6 (Citation Intensity Independence). It is easy to check that all other conditions hold.

\section{Characterizations of $\succsim_{c}$}

\section{$5.1 \quad$ Result}

We already observed that the $\mathrm{BRF} \succsim_{c}$ satisfies all conditions in Theorem 1, except A6 (Citation Intensity Independence). The following condition is satisfied by $\succsim_{c}$ but is violated by $\succsim_{n}$. It says that the ranking of two authors is not altered if one paper of each of these two authors receives one additional citation, coming from uncited papers signed by a third author. This condition is clearly satisfied by $\succsim_{c}$. Indeed, if $G$ is the profile before the modification and $H$ is the profile after the modification, we clearly have that $f_{c}(a, H)=f_{c}(a, G)+1, f_{c}(b, H)=f_{c}(b, G)+1$ (while, $f_{c}(c, H)=f_{c}(c, G)$, for all $\left.c \in \mathcal{A} \backslash\{a, b\}\right)$.

A7 (Independence of Added Citations) Let $G=(\mathcal{P}, S, C)$. Let $a, b, c \in \mathcal{A}$ be distinct authors and take $r, s \in \mathcal{P}$ such that $I C_{G}(r)=I C_{G}(s)=\varnothing, c S r$, and $c S$ s. Take $p \in P_{G}(a)$ and $q \in P_{G}(b)$. Consider now the profile $H=\left(\mathcal{P}, S, C^{*}\right)$ that is identical to $G$ except that $C^{*} \backslash C=\{(r, p),(s, q)\}$. Then, we have $a \succsim(G)$ $b \Leftrightarrow a \succsim(H) b$.

Replacing A6 (Citation Intensity Independence) with A7 (Independence of Added Citations) in Theorem 1 leads to a result characterizing $\succsim_{c}$.

\section{Theorem 2}

$A \mathrm{BRF} \succsim$ on $\mathcal{A}$ satisfies conditions $A 1-A 5$ and $A 7$ iff $\succsim=\succsim_{\text {c }}$

The necessity of the six conditions was discussed above. The proof of sufficiency is given in the next subsection. As above, we will also show that the above conditions are independent. 


\subsection{Sufficiency proof of Theorem 2}

Let us consider a bibliometric profile $G=(\mathcal{P}, S, C)$ on $\mathcal{A}$. Take $a, b \in \mathcal{A}$ with $a \neq b$. Because we have supposed that $|\mathcal{A}| \geq 3$, let us choose $c \in \mathcal{A} \backslash\{a, b\}$.

Step 1. We build a profile $G_{1}$ in which each paper is either a sender or a receiver. In $G_{1}$, all senders are signed by $c$ and cite a unique receiver. All receivers are signed either by $a$ or by $b$ and are cited by a unique sender.

Because we have supposed that the citation graph is without any cycle, there is a paper $p \in \mathcal{P}$ such that $I C_{G}(p)=\varnothing$.

If $O C_{G}(p)=\varnothing$, paper $p$ is a dummy paper. Using A3 (Dummy Paper), we can suppress it from $\mathcal{P}$.

If $p$ does not cite any paper signed by $a$ or by $b$, we can use A5 (Independence of Irrelevant Citations) to remove all citations emanating from $p$. The paper $p$ becomes a dummy paper that can be suppressed, using A3 (Dummy Paper).

Suppose that among the papers cited by $p$ there is a paper $q$ signed by $a$. For definiteness, suppose that the author of $p$ is $d \in \mathcal{A}$.

We will show that the citation from $p$ to $q$ may be deleted and replaced by a citation from a paper $p^{\prime}$ signed by $c$ to a paper $q^{*}$ signed by $a$. The paper $p^{\prime}$ will be a sender citing a single paper $\left(q^{*}\right)$. The paper $q^{*}$ will be a receiver cited by a single paper $\left(p^{\prime}\right)$.

Using A3 (Dummy Paper), we create four dummy papers $p^{*}$ and $p^{* *}$ signed by $d, q^{*}$ signed by $a$, and $q^{* *}$ signed by $b$. Using A7 (Independence of Added Citations) we add a citation from $p^{*}$ to $q^{*}$ and a citation from $p^{* *}$ to $q^{* *}$. Using A7 (Independence of Added Citations), we can suppress the citation from $p$ to $q$ and the citation from $p^{* *}$ to $q^{* *}$. The two papers $p^{* *}$ and $q^{* *}$ become dummy papers and can be suppressed, using A3 (Dummy Paper).

At this stage, we have replaced the citation from $p$, signed by $d$, to $q$, signed by $a$, by a citation from $p^{*}$, signed by $d$, to $q^{*}$, signed by $a$.

Using A3 (Dummy Paper), we now create two dummy papers $p^{\prime}$ and $q^{\prime}$ signed by $c$. Using A5 (Independence of Irrelevant Citations), we can add a citation from $p^{\prime}$ to $q^{\prime}$ without changing the position of $a$ w.r.t. $b$ in the ranking. We then use A4 (Restricted Source Independence) to exchange the citation from $p^{*}$ to $q^{*}$ with the citation from $p^{\prime}$ to $q^{\prime}$, without altering the ranking. Using A5 (Independence of Irrelevant Citations), the citation from $p^{*}$, signed by $d$, to $q^{\prime}$, signed by $c$, can be suppressed without altering the ranking of $a$ w.r.t. $b$. Once this is done, $p^{*}$ and $q^{\prime}$ become dummy papers and can be suppressed, using A3 (Dummy Paper).

Summarizing, we have replaced the citation from $p^{*}$, signed by $d$, to $q^{*}$, signed by $a$, by a citation from $p^{\prime}$, signed by $c$, to $q^{*}$, signed by $a$. Note that, in this process, the paper $q$ does not necessarily become a dummy paper because it is not excluded that it was cited by more than one paper in the original profile. It will 
become a dummy paper when all the papers citing it and belonging to the original set of papers will have been processed.

We can repeat this process for all papers cited by $p$ that are signed by $a$. Clearly the same operations can be performed for all papers cited by $p$ that are signed by $b$.

In the end, the paper $p$ will only cite papers not signed by $a$ or by $b$. All these citations can be suppressed, using A5 (Independence of Irrelevant Citations). After this is done, the paper $p$ becomes a dummy paper and can be suppressed.

The new citation graph that we have built is still without any cycle. It is easy to check that in this graph there must exist a paper from the original set of papers that is uncited. We then repeat the above operations for this uncited paper. We do so until we have processed all papers in the original set of papers. This defines the profile $G_{1}$. The ranking of $a$ w.r.t. $b$ in $G_{1}$ is the same as in $G$.

Notice that in $G_{1}$, a paper is either a sender (cites other papers but is not cited) or a receiver (is cited by other papers but does not cite any paper). By construction, all senders are signed by $c$. A sender cites a unique receiver. The receivers are cited by a unique sender. All receivers are signed either by $a$ or by $b$.

Suppose now that, in the initial profile $G$, we had $f_{c}(a, G)=f_{c}(b, G)$. Let us show that we must have $a \sim(G) b$.

It is easy to see that, in the profile $G_{1}$, the total number of citations received by papers signed by $a$ is equal to the total number of citations received by papers signed by $b$.

Step 2. Repeatedly using A7 (Independence of Added Citations), we can remove from $G_{1}$ all citations to papers signed by $a$ and to papers signed by $b$. This does not alter the ranking of $a$ w.r.t. $b$. After this is done, all papers become dummy papers. We use A3 (Dummy Paper) to suppress them. This creates the profile $G_{2}$ in which the set of papers is empty. The ranking of $a$ w.r.t. $b$ in $G_{2}$ is the same as in $G_{1}$.

Using A1 (Author Anonymity), we can then exchange the papers signed by $a$ with the papers signed by $b$ in $G_{2}$. Since these two authors do not sign any paper, the resulting profile is identical to $G_{2}$. This shows that, in $G_{2}, a$ and $b$ must belong to the same equivalence class. Hence, in $G$ also, $a$ and $b$ must belong to the same equivalence class.

Suppose now that, in the initial profile $G$, we had $f_{c}(a, G)>f_{c}(b, G)$. Let us show that we must have $a \succ(G) b$, which will complete the proof. 
It is easy to see that, in the profile $G_{1}$, the total number of citations received by papers signed by $a$ is strictly larger than the total number of citations received by papers signed by $b$.

Step 3. Using exactly the same reasoning as in Step 7 of the proof of Theorem 1 shows that we must have $a$ ranked strictly above $b$.

This completes the proof.

\subsection{Independence of the conditions in Theorem 2}

We give examples showing that the conditions used in Theorem 2 are independent.

Example 7 (A1 (Author Anonymity))

Choose $z \in \mathcal{A}$. Consider the BRF such that, for all bibliometric profiles $G$ on $\mathcal{A}$, and all $a, b \in \mathcal{A}$,

$$
a \succsim(G) b \Leftrightarrow g(a, G) \geq g(b, G)
$$

with

$$
g(a, G)=\left\{\begin{aligned}
f_{c}(a, G) & \text { if } a \neq z \\
2 f_{c}(a, G) & \text { if } a=z
\end{aligned}\right.
$$

Condition A1 (Author Anonymity) is violated. It is easy to check that all other conditions hold.

We know that the BRF in Example 2 satisfies A1 (Author Anonymity), A3 (Dummy Paper), A4 (Restricted Source Independence), and A5 (Independence of Irrelevant Citations) but violates A2 (Strong Monotonicity). It is easy to check that it satisfies A7 (Independence of Added Citations).

Example 8 (A3 (Dummy Paper))

Consider the BRF such that, for all bibliometric profiles $G$ on $\mathcal{A}$, and all $a, b \in \mathcal{A}$,

$$
a \succsim(G) b \Leftrightarrow g(a, G) \geq g(b, G)
$$

with

$$
g(a, G)=f_{c}(a, G)+\left|P_{G}(a)\right| .
$$

It is clear that this BRF violates A3 (Dummy Paper). All other conditions trivially hold.

Example 9 (A4 (Restricted Source Independence))

Consider the BRF such that, for all bibliometric profiles $G$ on $\mathcal{A}$, and all $a, b \in \mathcal{A}$,

$$
a \succsim(G) b \Leftrightarrow g(a, G) \geq g(b, G)
$$


with

$$
g(a, G)=\sum_{p \in P_{G}(a)}\left[\left|I C_{G}^{\prime}(p)\right|+\frac{1}{2}\left|I C_{G}^{\prime \prime}(p)\right|\right] .
$$

where, as before,

$$
\begin{aligned}
& I C_{G}^{\prime}(p)=\left\{q \in \mathcal{P}: q C p \text { and } S^{-1}(q) \neq S^{-1}(p)\right\}, \\
& I C_{G}^{\prime \prime}(p)=\left\{q \in \mathcal{P}: q C p \text { and } S^{-1}(q)=S^{-1}(p)\right\} .
\end{aligned}
$$

This BRF violates A4 (Restricted Source Independence). For instance, let $\mathcal{A}=$ $\{a, b, c\}$ and $G=(\mathcal{P}, S, C)$ a bibliometric profile on $\mathcal{A}$ with $\mathcal{P}=\left\{p, q, r, p^{*}, q^{*}, r^{*}\right\}$. Suppose that the signing relation $S$ is such that: $P_{G}(a)=\left\{p, q^{*}, r^{*}\right\}, P_{G}(b)=\left\{p^{*}\right\}$, and $P_{G}(c)=\{q, r\}$. Suppose finally that the citing relation $C$ is such that: $q C p$, $r C p, q^{*} C p^{*}, r^{*} C p^{*}$. It is easy to check that we have $g(a, G)=2$ and $g(b, G)=2$, so that $a \sim(G) b$.

Consider now the profile $H=\left(\mathcal{P}, S, C^{*}\right)$ that is identical to $G$, except that the citation relation is now such that: $q^{*} C^{*} p, r C^{*} p, q C^{*} p^{*}, r^{*} C^{*} p^{*}$. It is easy to check that we have $g(a, G)=1.5$ and $g(b, G)=2$, so that $b \succ(G) a$, violating A4 (Restricted Source Independence).

It is simple to check that all other conditions hold. For instance, A7 (Independence of Added Citations) holds, since in this condition, the additional citations to papers signed by $a$ and $b$ always come from papers signed by a third author $c$. $\diamond$

Example 10 (A5 (Independence of Irrelevant Citations))

Take a profile $G=(\mathcal{P}, S, C)$. Consider the BRF such that, for all bibliometric profiles $G$ on $\mathcal{A}$, and all $a, b \in \mathcal{A}$,

$$
a \succsim(G) b \Leftrightarrow g(a, G) \geq g(b, G)
$$

with

$$
\left.g(a, G)=\sum_{p \in P_{G}(a)}\left[\left|I C_{G}(p)\right|-h(p, G)\right)\right] .
$$

where $h(p, G)$ is the function that was used in Example 5.

This BRF violates A5 (Independence of Irrelevant Citations). For instance, let $\mathcal{A}=\{a, b, c\}$ and $G=(\mathcal{P}, S, C)$ a bibliometric profile on $\mathcal{A}$ such that $\mathcal{P}=$ $\left\{\alpha^{1}, \alpha^{2}, \beta^{1}, \beta^{2}, \gamma, p, q\right\}$.

Suppose that the signing relation $S$ is such that:

$$
\begin{aligned}
& P_{G}(a)=\{p\} \\
& P_{G}(b)=\{q\} \\
& P_{G}(c)=\left\{\alpha^{1}, \alpha^{2}, \beta^{1}, \beta^{2}, \gamma\right\} .
\end{aligned}
$$


Suppose finally that the citing relation $C$ is such that:

$$
\begin{aligned}
& \alpha^{1} C p, \alpha^{2} C p, \\
& \beta^{1} C q, \beta^{2} C q .
\end{aligned}
$$

It is easy to check that we have $a \sim(G) b$. If we define the profile $H=\left(\mathcal{P}, S, C^{*}\right)$ that is identical to $G$, except that we have added a citation from $p$ to $\gamma$, we obtain $b \succ(H) a$.

It is not difficult to check that all other conditions are satisfied. For instance, A7 (Independence of Added Citations) is satisfied because this condition does not alter the number of papers signed by $a$ and by $b$ and leaves unchanged the citations that these papers give to other papers. Because the function $h(p, G)$ is always strictly less than 1 , the addition of a citation from a paper signed by $a$ to another paper signed by $a$ always improves $g(a, G)$. Hence, A2 (Strong Monotonicity) is always satisfied. A4 (Restricted Source Independence) is satisfied because this condition leaves unchanged the number of citations given and received by all papers.

Example 11 (A7 (Independence of Added Citations))

It is easy to check that the BRF $\succsim_{n}$ violates A7 (Independence of Added Citations). Theorem 1 has shown that all other conditions hold.

\section{Remark 3}

The characterization presented in Theorem 2 was designed with the constraint to use as many conditions already used in Theorem 1 as possible. Indeed, Theorems 1 and 2 both use six conditions and have five conditions in common. This comes at a price however. Indeed, the bibliometric ranking based on the number of citations is rather simple and more direct characterizations of $\succsim_{c}$ can be envisaged. For space reasons, we do not develop this point here.

\section{Discussion}

\subsection{Setting}

Most of the literature devoted to the formal properties of BRFs use a setting different from the one used here ${ }^{8}$. In these papers (see, e.g., Bouyssou and Marchant,

\footnotetext{
${ }^{8}$ The differences between our setting and the more classical one is reminiscent of the distinction between multi-profile and single-profile approaches in Social Choice Theory. A classic example of a multi-profile setting is Arrow (1963). An example a single-profile approach is Roberts (1980).

As pointed out to us by a referee, analogies with Social Choice Theory could be pushed even further. Handling authors from several fields is not unlike aggregating votes that are made in several electoral districts: votes (resp. citations) given in different districts (resp. fields) may not be given the same "worth".
} 
2011b, 2014, Chambers and Miller, 2014, Deineko and Woeginger, 2009, Hwang, 2013, Kongo, 2014, Marchant, 2009a,b, Miroiu, 2013, Quesada, 2009, 2010, 2011a,b, Woeginger, 2008a,b) an author $a$ is viewed as a mapping from $\mathbb{N}$ to $\mathbb{N}$. For $x \in \mathbb{N}$, the value $a(x)$ is the number of publications of author $a$ with exactly $x$ citations. The set of all authors is then the set of all such functions $a$ for which $\sum_{x \in \mathbb{N}} a(x)$ is finite.

In the setting used in the papers mentioned above, the set of authors is infinite and contains all possible conceivable authors. Moreover, it does not allow one to distinguish citations based on the name of citing paper or based on the name of the author of the citing paper. This setting makes it impossible to formulate a condition like A4 (Restricted Source Independence). A condition resembling the weakening of condition A2 (Strong Monotonicity) evoked in Section 3 and requiring that if $a$ is judged not worse than $b$, it remains so after the addition of a citation to a paper signed by $a$, is taken to be part of the definition of a bibliometric ranking in most texts (see, e.g., Bouyssou and Marchant, 2014, Woeginger, 2008a). The conditions corresponding to A1 (Author Anonymity), A3 (Dummy Paper) and A5 (Independence of Irrelevant Citations) reformulated in this setting are not automatically satisfied. However, in this setting, BRFs that violate them are quite contrived.

The framework that we use here (that is a simplified version of the one used in Bouyssou and Marchant, 2010) is more detailed and it allows conditions A1 (Author Anonymity), A3 (Dummy Paper), A4 (Restricted Source Independence), and A5 (Independence of Irrelevant Citations) to have a real bite. It is more complex than the usual setting but is also probably more realistic: when we look at a bibliometric database, what we have is a given profile $G$ and not the set of all potential authors.

\subsection{Conditions}

Our two main results both use six conditions. They have in common five conditions A1 (Author Anonymity), A2 (Strong Monotonicity), A3 (Dummy Paper), A4 (Restricted Source Independence), and A5 (Independence of Irrelevant Citations). Let us now analyze in more detail these conditions in our setting.

Condition A1 (Author Anonymity) seems unavoidable. Abandoning it would mean that some authors have an advantage because they possess a distinctive characteristic feature (e.g., their name). This would be the case with a BRF always ranking authors according to the lexicographic order of their last name. Needless to say that such BRFs have little to bring to the field. We are not aware of any sensible BRF that would violate this condition.

Condition A2 (Strong Monotonicity) is a rather stringent monotonicity condition. It says that adding one adequate citation is always sufficient to break a tie in 
the expected direction. A bibliometric ranking based on the $h$-index (Hirsch, 2005) or on the number of papers signed by an author (Marchant, 2009a) would violate it. Its weakening evoked in Section 3 seems, on the contrary, rather innocuous.

Condition A3 (Dummy Paper) is satisfied by most BRFs that are of common use. The only classical BRF that violates this condition is the one based on the number of papers. The limitations of such a way of ranking authors should be clear.

Condition A4 (Restricted Source Independence) seems rather innocuous. It is satisfied by all BRFs that are of common use (e.g., all those studied in Bouyssou and Marchant, 2014). It is violated by ranking methods in which self-citations are not treated as ordinary citations.

Condition A5 (Independence of Irrelevant Citations) also seems innocuous. Indeed, it is satisfied by all BRFs that are of common use (e.g., all those studied in Bouyssou and Marchant, 2014). It is violated by ranking methods in which the position of the author of the citing paper in the ranking has an influence on the value of citations made, e.g., ranking methods in which a citation coming from a highly ranked author has more "value" than a citation coming a lowly ranked author (see, e.g., West, Jensen, Dandrea, Gordon, and Bergstrom, 2013). This includes all BRFs à la PageRank. The application of such recursive ranking methods have been the subject of many studies in the recent years (see Fragkiadiki and Evangelidis, 2014, Waltman and Yan, 2014, for overviews). The case of the evaluation of journals has received special attention (Demange, 2014, PalaciosHuerta and Volij, 2004, Pinski and Narin, 1976).

Hence, the five conditions that are common to our two results appear rather reasonable. Let us now turn to the two remaining conditions.

Condition A6 (Citation Intensity Independence) is probably the most complex and the least simple to justify. We like to think of it in the following way. Suppose that the editor of a journal requires all papers published in this journal to cite not only the published version of the cited papers but also their last working paper version on a given Working Paper Repository ${ }^{9}$. It is difficult to imagine why such a requirement, however weird, should influence the ranking of authors. Our condition A6 (Citation Intensity Independence) with $k=1$ requires less, since it only applies to uncited papers. The requirement of the editor of the journal may be interpreted as a local increase in citation intensity. All papers published in this journal will double the number of citations they make. A condition saying that this should have have no impact on the ranking of all authors does not seem unreasonable.

To motivate Condition A6 (Citation Intensity Independence) for all $k \in \mathbb{N}_{+}$

\footnotetext{
${ }^{9}$ E.g., arXiv (http://arxiv.org/), RePEc (http://repec.org/), or SSRN (http://www . ssrn. com/).
} 
(and not just for $k=1$ as in the previous example), we can think of the following situation. Suppose author $a$ writes paper $p$ and considers she has to cite papers $q^{1}, q^{2}, q^{3}$, and $q^{4}$ because all four are essential for a good understanding of paper $p$ and of its contribution to the field. Suppose now, for all $j \in\{1,2,3,4\}$, that $b^{j}$ (the author of $q^{j}$ ) has also written $q_{1}^{j}, q_{2}^{j}$ and $q_{3}^{j}$ on the same topic as $q^{j}$. Author a considers $q_{1}^{j}, q_{2}^{j}$ and $q_{3}^{j}$ as relevant, but to a lesser extent. Depending on the tradition in his research domain, author $a$ will cite only $q^{1}, q^{2}, q^{3}$, and $q^{4}$ (profile $G$ ) or those four plus some of the papers $q_{i}^{j}$.

If $a$ cites $q^{1}, q^{2}, q^{3}, q^{4}$, and $q_{1}^{1}$ (profile $G^{*}$ ), the local citation intensity in the local field around $p$ increases, but it is also clear that the position of $b_{1}$ in $G^{*}$ improves with respect to the position of $b_{2}, b_{3}$, and $b_{4}$. And if $\succsim(G) \neq \succsim\left(G^{*}\right)$, we then have no clear reason to object.

Suppose now a cites $q^{1}, q^{2}, q^{3}, q^{4}$ and $q_{i}^{1}, q_{i}^{2}, q_{i}^{3}, q_{i}^{4}$ for all $i \in\{1,2,3\}$ (profile $G^{*}$ ). The relative position of $b_{1}, b_{2}, b_{3}$, and $b_{4}$ does not change, because of the symmetry in the change from $G$ to $G^{*}$. So the ranking of these three authors should be the same in $\succsim(G)$ and in $\succsim\left(G^{*}\right)$. Since the only change in the local field around $p$ is the increase in citation intensity, we can also argue (as in the example of the working paper repository) that the position of $b_{1}$ (or $b_{2}$ or $b_{3}$ or $b_{4}$ ) should not change with respect to the position of any other author. In conclusion, we should have $\succsim(G)=\succsim\left(G^{*}\right)$.

Although A6 (Citation Intensity Independence) is not unreasonable, it is complex. It would be nice to factorize it into simpler conditions that would be easier to grasp.

We know that $\succsim_{c}$ violates this requirement. It is easy to find examples showing that it is also violated by the ranking based on the $h$-index or on the number of highly cited papers.

Condition A7 (Independence of Added Citations) is satisfied by many BRFs, including some BRFs that have a recursive character. Indeed, with this condition, we add to both authors $a$ and $b$ a citation coming from a paper signed by the same author $c$. We nevertheless saw that this condition is violated by $\succsim_{n}$ because the papers $r$ and $s$ both signed by $c$ that are both giving an additional citation may cite a different number of papers and, thus, may count differently using $\succsim_{n}$. It is also easy to check that it is violated by the ranking based on the $h$-index (Hirsch, 2005) or on the number of highly cited papers (as defined, e.g., in Bouyssou and Marchant, 2014, Waltman and van Eck, 2012b).

\subsection{Directions for future research}

This paper has studied bibliometric rankings but has not considered bibliometric indices. The study of the index $f_{n}$ seems interesting. One may think that going from rankings to indices is not difficult. Indeed, Bouyssou and Marchant (2014) 
have shown that, whenever two consecutive equivalence classes of the ranking are always "equally spaced" in terms of the index, a characterization of the index is easily derived from a characterization of the ranking. This is not so here, for at least two reasons. First, in our setting, the profile $G$ under study may not contain authors belonging to all consecutive equivalence classes, contrary to what happens when the set of authors contains all possible conceivable authors (as in Bouyssou and Marchant, 2014). Second, even if this could be ensured, consecutive equivalence classes of $\succsim_{n}$ are not equally spaced in terms of $f_{n}$. Hence the axiomatic study of $f_{c}$ would require further research. We think that the analysis developed in this paper may nevertheless be useful for doing so.

With $\succsim_{n}$, the weight of a citation given by a paper $q$ is inversely proportional to $\left|O C_{G}(q)\right|$. Whereas it seems sensible to choose a weight that decreases with $\left|O C_{G}(q)\right|$, the choice of this particular decreasing function is clearly more open to debate. In fact, the BRF $\succsim_{n}$ is a member of a more general family of BRFs in which the weight of a citation given by paper $q$ is equal to $w\left(\left|O C_{G}(q)\right|\right)$, with $w$ a nonincreasing function from $\mathbb{N}_{+}$to the nonnegative reals. A study of this family of rankings is a research question that seems promising.

Other variants can be considered. Consider, e.g., two papers $q$ and $q^{*}$ giving the same number of citations. With the BRF $\succsim_{n}$, all these citations have the same weight. This seems open to discussion if, e.g., we know that $q$ is cited by many papers but that $q^{*}$ is not. In such a case, we would like the citations given by $q$ to have more weight. This suggests another family of BRFs in which the weight of a citation given by $q$ would be equal to $w^{*}\left(\left|I C_{G}(q)\right|,\left|O C_{G}(q)\right|\right)$ where $w^{*}$ would be nondecreasing in its first argument and nonincreasing in its second argument.

Another potentially interesting extension would be to use $f_{n}$ "recursively", e.g., using a ranking that would be based on the following index:

$$
f_{n}^{(2)}(a, G)=\sum_{p \in P_{G}(a)}\left[\sum_{q \in I C_{G}(p)} \frac{f_{n}\left(S^{-1}(q), G\right)}{\left|O C_{G}(q)\right|}\right],
$$

in which the "value" of an author $a$ is first computed as $f_{n}(a, G)$ and then each paper signed by $a$ divides this value equally among all the papers that it $\operatorname{cites}^{10}$. This would bring us closer to the rankings à la PageRank (see, for the case of journals, Demange, 2014, Palacios-Huerta and Volij, 2004, Pinski and Narin, 1976; see also Slutzki and Volij, 2005, 2006).

\footnotetext{
${ }^{10}$ One may think of distributing, instead of the value associated with the author, the value associated with each paper, as given by $f_{n}$. Because the citation graph is without any cycle, this does not work without some adaptation. Indeed, papers that are uncited will have a null value and this null value will propagate to the whole set of papers. A possible solution to this problem, as suggested to us by a referee, is to associate a minimal positive to each paper on top of the value given by $f_{n}$, that could be interpreted as the "value of being published".
} 


\subsection{Final comments}

We mentioned in Section 1 that there are many different ways to perform a fieldnormalization, keeping in line with the idea of "citing-side" normalization. We have only investigated one of them. Furthermore, we have concentrated on the formal properties of the ranking induced by the index $f_{n}$ and we have mentioned that our setting conceals many important empirical problems (choice of an adequate time window, choice of the "core journals", in the sense of Waltman and van Eck (2013a), discussion of the criteria for judging the appropriateness of a given normalization, ease of computation of the underlying index, avoidance of potential manipulation of the index by authors and editors of journals, etc.). These quite important points are left open by the present paper.

In view of the heated discussions around the "crown indicator" used by the Leiden group (CWTS) (Bornmann, 2010, Bornmann and Mutz, 2011, Gingras and Larivière, 2011, Larivière and Gingras, 2011, Leydesdorff and Opthof, 2011a, Lundberg, 2007, Opthof and Leydesdorff, 2010, 2011, van Raan, van Leeuwen, Visser, van Eck, and Waltman, 2010, Waltman, van Eck, van Leeuwen, Visser, and van Raan, 2011a,b,c, Waltman, Calero-Medina, Kosten, Noyons, Tijssen, van Eck, van Leeuwen, van Raan, Visser, and Wouters, 2012a) and around the SNIP indicator (Leydesdorff, 2013, Leydesdorff and Opthof, 2011b, Mingers, 2014, Moed, 2010, 2011, Waltman et al., 2013), we nevertheless think that the discussion of the formal properties of a ranking method is a useful exercise. Indeed, intuitively appealing formulas giving empirically meaningful results may be plagued by disappointing behavior in certain situations. Axiomatic analyses like the one proposed here may help detecting such problems.

\section{References}

G. Abramo, T. Cicero, and C. A. D'Angelo. Revisiting the scaling of citations for research assessment. Journal of Informetrics, 6(4):470-479, 2012.

P. Albarrán, J. A. Crespo, I. Ortuño, and J. Ruiz-Castillo. The skewness of science in 219 sub-fields and a number of aggregates. Scientometrics, 88(2):385-397, 2011.

A. Altman. The PageRank axioms. In D. Lehmann, R. Müller, and T. Sandholm, editors, Computing and Markets, number 05011 in Dagstuhl Seminar Proceedings, Dagstuhl, Germany, 2005. Internationales Begegnungs- und Forschungszentrum für Informatik (IBFI), Schloss Dagstuhl, Germany.

A. Altman and M. Tennenholtz. Ranking systems: the PageRank axioms. In Proceedings 6th ACM Conference on Electronic Commerce (EC-2005), Vancouver, BC, Canada, June 5-8, 2005, pages 1-8, 2005.

M. Amin and M. A. Mabe. Impact factors: Use and abuse. Perspectives in Publishing, 1:1-6, 2000. 
K. J. Arrow. Social choice and individual values. Wiley, New York, 2nd edition, 1963.

L. Bornmann. Towards an ideal method of measuring research performance: Some comments to the Opthof and Leydesdorff (2010) paper. Journal of Informetrics, 4: 441-443, 2010.

L. Bornmann. How to analyze percentile citation impact data meaningfully in bibliometrics: The statistical analysis of distributions, percentile rank classes, and top-cited papers. Journal of the American Society for Information Science E Technology, 64(3): 587-595, 2013.

L. Bornmann and H.-D. Daniel. Universality of citation distributions-A validation of Radicchi et al.'s relative indicator $c_{f}=c / c_{0}$ at the micro level using data from Chemistry. Journal of the American Society for Information Science \& Technology, 60(8): 1664-1670, 2009.

L. Bornmann and W. Marx. Methods for the generation of normalized citation impact scores in bibliometrics: Which method best reflects the judgements of experts? Journal of Informetrics, 9:408-418, 2015.

L. Bornmann and R Mutz. Further steps towards an ideal method of measuring citation performance: The avoidance of citation (ratio) averages in field normalization. Journal of Informetrics, 5:228-230, 2011.

L. Bornmann, L. Leydesdorff, and R. Mutz. The use of percentiles and percentile rank classes in the analysis of bibliometric data: Opportunities and limits. Journal of Informetrics, 7(1):158-165, 2013.

D. Bouyssou. Monotonicity of 'ranking by choosing' procedures: A progress report. Social Choice and Welfare, 23(2):249-273, 2004.

D. Bouyssou and T. Marchant. Consistent bibliometric rankings of authors and journals. Journal of Informetrics, 4(3):365-378, 2010.

D. Bouyssou and T. Marchant. Bibliometric rankings of journals based on impact factors: An axiomatic approach. Journal of Informetrics, 5(1):75-86, 2011a.

D. Bouyssou and T. Marchant. Ranking scientists and departments in a consistent manner. Journal of the American Society for Information Science \& Technology, 62(9): 1761-1769, 2011b.

D. Bouyssou and T. Marchant. An axiomatic approach to bibliometric rankings and indices. Jouranl of Informetrics, 8(3):449-477, 2014.

R. van den Brink and R. F. Gilles. Measuring domination in directed networks. Social networks, 22:141-157, 2000.

R. van den Brink and R. F. Gilles. Digraph competitions and cooperative games. Theory and Decision, 53:327-342, 2002.

C. P. Chambers and A. D. Miller. Scholarly influence. Journal of Economic Theory, 151:571-583, 2014.

J. A. Crespo, I. Ortuño-Ortín, and J. Ruiz-Castillo. The citation merit of scientific publications. PLoS ONE, 7(11):e49156, 11 2012. doi: http://dx.doi.org/10.1371\%2Fjournal. pone.0049156.

J. A. Crespo, Y. Li, and J. Ruiz-Castillo. The measurement of the effect on citation inequality of difference in citation practices accross scientific fields. PLOS ONE, 8(3): 
e58727, 2013.

J. A. Crespo, N. Herranz, Y. Li, and J. Ruiz-Castillo. The effect on citation inequality of differences in citation practices at the web of science subject category level. Journal of the Association for Information Science \& Technology, 65(6):1244-1256, 2014.

V. G. Deineko and G. J. Woeginger. A new family of scientific impact measures: The generalized Kosmulski-indices. Scientometrics, 80(3):819-828, 2009.

G. Demange. A ranking method based on handicaps. Theoretical Economics, 9:915-942, 2014.

B. Dutta and J.-F. Laslier. Comparison functions and choice correspondences. Social Choice and Welfare, 16:513-532, 1999.

N. J. van Eck, L. Waltman, A. F. J. van Raan, R. J. M. Klautz, and W. C. Peul. Citation analysis may severely underestimate the impact of clinical research as compared to basic research. PLOS ONE, 8(4):e62395, 2013.

E. Fragkiadiki and G. Evangelidis. Review of the indirect citations paradigm: theory and practice of the assessment of papers, authors and journals. Scientometrics, 99: 261-288, 2014.

F. Franceschini and D. Maisano. Sub-field normalization of the IEEE scientific journals based on their connection with technical societies. Journal of Informetrics, 8:508-533, 2014.

Y. Gingras and V. Larivière. There are neither "kings" or "crown" in scientometrics: comments on a supposed "alternative" method of normalization. Journal of Informetrics, 5:226-227, 2011.

W. Glänzel, A. Schubert, B. Thijs, and K. Debackere. A priori vs. a posteriori normalisation of citation indicators. The case of journal ranking. Scientometrics, 87(2): 415-424, 2011.

J. E. Hirsch. An index to quantify an individual's scientific research output. Proceedings of the National Academy of Sciences, 102(46):16569-16572, 2005.

Y.-A. Hwang. An axiomatization of the Hirsch-index without adopting monotonicity. Applied Mathematics \& Information Sciences. An International Journal, 7(4):13171322, 2013.

J. Kaur, E. Ferrara, F. Menczer, A. Flammini, and F. Radicchi. Quality versus quantity in scientific impact. Journal of Informetrics, 9(4):800-808, 2015. ISSN 1751-1577. doi: http://dx.doi.org/10.1016/j.joi.2015.07.008.

L. Á. Kóczy and M. Strobel. The ranking of economic journals by a tournament method. Working Paper, Maastricht University, 2007.

T. Kongo. An alternative axiomatization of the Hirsch index. Journal of Informetrics, 8(1):252-258, 2014.

V. Larivière and Y. Gingras. Averages of ratios vs. ratios of averages: An empirical analysis of four levels of aggregation. Journal of Informetrics, 5:392-399, 2011.

J.-F. Laslier. Tournament solutions and majority voting. Springer-Verlag, Berlin, 1997. L. Leydesdorff. The revised SNIP indicator of Elsevier's Scopus. Journal of Informetrics, $7(4): 859-860,2013$.

L. Leydesdorff and L. Bornmann. Percentile ranks and the integrated impact indicator 
(i3). Journal of the American Society for Information Science 83 Technology, 63(9): 1901-1902, 2012.

L. Leydesdorff and T. Opthof. Scopus's Source Normalized Impact per Paper (SNIP) versus a journal impact factor based on fractional counting of citations. Journal of the American Society for Information Science \& Technology, 61(11):2365-2369, 2010a.

L. Leydesdorff and T. Opthof. Normalization at the field level: Fractional counting of citations. Journal of Informetrics, 4(4):644-646, $2010 \mathrm{~b}$.

L. Leydesdorff and T. Opthof. Remaining problems with the "New Crown Indicator" (MNCS) of the CWTS. Journal of Informetrics, 5:224-225, 2011a.

L. Leydesdorff and T. Opthof. Scopus' SNIP indicator: Reply to Moed. Journal of the American Society for Information Science \& Technology, 62(1):214-215, 2011b.

L. Leydesdorff and J. C. Shin. How to evaluate universities in terms of their relative citation impacts: Fractional counting of citations and the normalization of differences among disciplines. Journal of the American Society for Information Science 83 Technology, 62(6):1146-1155, 2011.

L. Leydesdorff, L. Bornmann, R. Mutz, and T. Opthof. Turning the tables on citation analysis one more time: Principles for comparing sets of documents. Journal of the American Society for Information Science \& Technology, 62(7):1370-1381, 2011.

L. Leydesdorff, F. Radicchi, L. Bornmann, C. Castellano, and W. de Nooy. Fieldnormalized impact factors (IFs): A comparison of rescaling and fractionally counted IFs. Journal of the American Society for Information Science \& Technology, 64(11): 2299-2309, 2013a.

L. Leydesdorff, P. Zhou, and L. Bornmann. How can journal impact factors be normalized across fields of science? An assessment in terms of percentile ranks and fractional counts. Journal of the American Society for Information Science \& Technology, 64(1): 96-107, 2013b.

Y. Li and J. Ruiz-Castillo. The comparison of normalization procedures based on different classification systems. Journal of Informetrics, 7(4):945-958, 2013.

Y. Li, F. Radicchi, C. Castellano, and J. Ruiz-Castillo. Quantitative evaluation of alternative field normalization procedures. Journal of Informetrics, 7(3):746-755, 2013. J. Lundberg. Lifting the crown-citation $z$-score. Journal of Informetrics, 1:145-154, 2007.

T. Marchant. An axiomatic characterization of the ranking based on the h-index and some other bibliometric rankings of authors. Scientometrics, 80(2):327-344, 2009a.

T. Marchant. Score-based bibliometric rankings of authors. Journal of the American Society for Information Science \&3 Technology, 60:1132-1137, 2009b.

D. C. McGarvey. A theorem on the construction of voting paradoxes. Econometrica, 21 (4):608-610, 1953.

J. Mingers. Problems with the SNIP indicator. Journal of Informetrics, 8:890-894, 2014.

A. Miroiu. Axiomatizing the Hirsch index: Quantity and quality disjoined. Journal of Informetrics, 7(1):10-15, 2013.

H. F. Moed. Measuring contextual citation impact of scientific journals. Journal of Informetrics, 4(3):265-277, 2010. 
H. F. Moed. The Source Normalized Impact per Paper is a valid and sophisticated indicator of journal citation impact. Journal of the American Society for Information Science \& Technology, 62(1):211-213, 2011.

T. Opthof and L. Leydesdorff. Caveats for the journal and field normalizations in the CWTS ("Leiden") evaluations of research performance. Journal of Informetrics, 4: 423-430, 2010.

T. Opthof and L. Leydesdorff. A comment on the paper by Waltman et al., Scientometrics, 87, 467, 2011. Scientometrics, 88:1011-1016, 2011.

L. Page and S. Brin. The anatomy of a large-scale hypertextual Web search engine. Computer Networks and ISDN Systems, 30(1-7):107-117, 1998.

I. Palacios-Huerta and O. Volij. The measurement of intellectual influence. Econometrica, 72:963-978, 2004.

A. Perianes-Rodriguez and J. Ruiz-Castillo. Multiplicative versus fractional counting methods for co-authored publications. the case of the 500 universities in the leiden ranking. Journal of Informetrics, 9(4):974-989, 2015. doi: http://dx.doi.org/10.1016/ j.joi.2015.10.002.

G. Pinski and F. Narin. Citation influence for journal aggregates of scientific publications: Theory, with applications to the literature of physics. Information Processing and Management, 12:297-312, 1976.

A. Quesada. Monotonicity and the Hirsch index. Journal of Informetrics, 3(2):158-160, 2009 .

A. Quesada. More axiomatics for the Hirsch index. Scientometrics, 82:413-418, 2010.

A. Quesada. Axiomatics for the Hirsch index and the Egghe index. Journal of Informetrics, 5:476-480, 2011a.

A. Quesada. Further characterizations of the Hirsch index. Scientometrics, 87:107-114, 2011b.

A. F. J. van Raan. Measuring science: Capita selecta of current main issues. In H. F. Moed, W. Glänzel, and U. Schmoch, editors, Handbook of Quantitative Science and Technology Research, pages 19-50, 2004.

A. F. J. van Raan. Measurement of central aspects of scientific research: Performance, interdisciplinarity, structure. Measurement: Interdisciplinary Research and Perspectives, $3(1): 1-19,2005$.

A. F. J. van Raan. Comparison of the Hirsch-index with standard bibliometric indicators and with peer judgment for 147 chemistry research groups. Scientometrics, 67:491-502, 2006.

A. F. J. van Raan, T. N. van Leeuwen, M. S. Visser, N. J. van Eck, and L. Waltman. Rivals to the crown: Reply to Opthof and Leydesdorff. Journal of Informetrics, 4: 431-435, 2010.

F. Radicchi and C. Castellano. Testing the fairness of citation indicators for comparison across scientific domains: The case of fractional citation counts. Journal of Informetrics, 6(1):121-130, 2012a.

F. Radicchi and C. Castellano. A reverse engineering approach to the suppression of citation biases reveals universal properties of citation distributions. PLOS ONE, 7(3): 
e33833, 2012b.

F. Radicchi, S. Fortunato, and C. Castellano. Universality of citation distributions: Toward an objective measure of scientific impact. Proceeding of the National Academy of Sciences, 105(45):17268-17272, 2008.

K. W. S. Roberts. Social choice theory: Single-profile and multi-profile approaches. Review of Economic Studies, 47:441-450, 1980.

A. Rubinstein. Ranking the participants in a tournament. SIAM Journal of Applied Mathematics, 38:108-111, 1980.

J. Ruiz-Castillo. The evaluation of citation distributions. SERIEs, 3:291-310, 2012.

J. Ruiz-Castillo. The comparison of classification-system-based normalization procedures with source normalization alternative in Waltman and Van Eck (2013). Journal of Informetrics, 8:25-28, 2014.

J. Ruiz-Castillo and R. Costas. The skewness of scientific productivity. Journal of Informetrics, 8(4):917-934, 2014. ISSN 1751-1577. doi: http://dx.doi.org/10.1016/j. joi.2014.09.006.

J. Ruiz-Castillo and L. Waltman. Field-normalized citation impact indicators using algorithmically constructed classification systems of science. Journal of Informetrics, 9 (1):102-117, 2015.

A. Schubert and T. Braun. Relative indicators and relational charts for comparative assessment of publication output and citation impact. Scientometrics, 9(5-6):281-291, 1986.

A. Schubert and T. Braun. Cross-field normalization of scientometric indicators. Scientometrics, 36(3):311-324, 1996.

T. Schwartz. Rationality and the myth of the maximum. Noûs, 6:97-117, 1972.

D. Sirtes. Finding the Easter eggs hidden by oneself: Why Radicchi and Castellano's (2012) fairness test for citation indicators is not fair. Journal of Informetrics, 6:448-450, 2012.

G. Slutzki and O. Volij. Ranking participants in generalized tournaments. International Journal of Game Theory, 33:255-270, 2005.

G. Slutzki and O. Volij. Scoring of web pages and tournaments - axiomatizations. Social Choice and Welfare, 26:75-92, 2006.

H. Small and E. Sweeney. Clustering the Science Citation Index ${ }^{\circledR}$ using co-citations. I. A comparison of methods. Scientometrics, 7(3-6):391-409, 1985.

L. Waltman and N. J. van Eck. A new methodology for constructing a publicationlevel classification system of science. Journal of the American Society for Information Science $\&$ Technology, 63(12):2378-2392, 2012a.

L. Waltman and N. J. van Eck. The inconsistency of the $h$-index. Journal of the American Society for Information Science \& Technology, 63(2):406-415, 2012b.

L. Waltman and N. J. van Eck. A systematic empirical comparison of different approaches for normalizing citation impact indicators. Journal of Informetrics, 7(4):833849, 2013a.

L. Waltman and N. J. van Eck. Source normalized indicators of citation impact: an overview of different approaches and an empirical comparison. Scientometrics, 96(3): 
699-716, 2013b.

L. Waltman and N. J. van Eck. Field-normalized citation impact indicators and the choice of an appropriate counting method. Working paper, arXiv:1501.04431, CWTS, Leiden, 2015.

L. Waltman and E. Yan. PageRank-related methods for analyzing citation networks. In Y. Ding, R. Rousseau, and D. Wolfram, editors, Measuring Scholarly Impact, pages 83-100. Springer International Publishing, 2014.

L. Waltman, N. J. van Eck, and E. C. M. Noyons. A unified approach to mapping and clustering of bibliometric networks. Journal of Informetrics, 4(4):629-635, 2010.

L. Waltman, N. J. van Eck, T. N. van Leeuwen, M. S. Visser, and A. F. J. van Raan. On the correlation between bibliometric indicators and peer review: reply to Opthof and Leydesdorff. Scientometrics, 88:1017-1022, 2011a.

L. Waltman, N. J. van Eck, T. N. van Leeuwen, M. S. Visser, and A. F. J. van Raan. Towards a new crown indicator: An empirical analysis. Scientometrics, 87(3):467-481, 2011b.

L. Waltman, N. J. van Eck, T. N. van Leeuwen, M. S. Visser, and A. F. J. van Raan. Towards a new crown indicator: Some theoretical considerations. Journal of Informetrics, 5(1):37-47, 2011c.

L. Waltman, C. Calero-Medina, J. Kosten, E. C. M. Noyons, R. J. W. Tijssen, N. J. van Eck, T. N. van Leeuwen, A. F. J. van Raan, M. S. Visser, and P. Wouters. The Leiden ranking 2011/2012: Data collection, indicators, and interpretation. Journal of the American Society for Information Science 83 Technology, 63(12):2419-2432, 2012a.

L. Waltman, N. J. van Eck, and A. F. J. van Raan. Universality of citation distributions revisited. Journal of the American Society for Information Science 83 Technology, 63 (1):72-77, 2012b.

L. Waltman, N. J. van Eck, T. N. van Leeuwen, and M. S. Visser. Some modifications to the SNIP journal impact indicator. Journal of Informetrics, 7(2):272-285, 2013.

J. D. West, M. C. Jensen, R. J. Dandrea, G. J. Gordon, and C. T. Bergstrom. Authorlevel Eigenfactor metrics: Evaluating the influence of authors, institutions, and countries within the Social Science Research Network community. 64(4):787-801, 2013.

G. J. Woeginger. An axiomatic characterization of the Hirsch-index. Mathematical Social Sciences, 56:224-232, 2008a.

G. J. Woeginger. A symmetry axiom for scientific impact indices. Journal of Informetrics, 2(4):298-303, 2008b.

H. P. Young. An axiomatization of Borda's rule. Journal of Economic Theory, 9:43-52, 1974.

P. Zhou and L. Leydesdorff. Fractional counting of citations in research evaluation: A cross- and interdisciplinary assessment of the Tsinghua university in Beijing. Journal of Informetrics, 5(3):360-368, 2011.

M. Zitt. Citing-side normalization of journal impact: A robust variant of the Audience Factor. Journal of Informetrics, 4(3):392-406, 2010.

M. Zitt. Behind citing-side normalization of citations: Some properties of the journal impact factor. Scientometrics, 89(1):329-344, 2011. 
M Zitt and J.-P. Cointet. Citation impacts revisited: A window on inter-disciplinarity and structural change in science networks. Working paper, INRA-Lereco, 2014.

M. Zitt and H. Small. Modifying the Journal Impact Factor by fractional citation weighting: The Audience Factor. Journal of the American Society for Information Science 85 Technology, 59(11):1856-1860, 2008.

M Zitt, S Ramanana-Rahary, and E Bassecoulard. Relativity of citation performance and excellence measures: From cross-field to cross-scale effects of field-normalisation. Scientometrics, 63(2):373-401, 2005. 